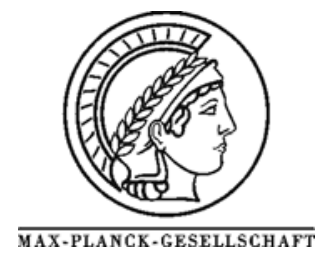

\title{
Strained thin copper films as model catalysts in the materials gap
}

\author{
F. Girgsdies ${ }^{\mathrm{a}}$, T. Ressler ${ }^{\mathrm{a},}$, U. Wild ${ }^{\mathrm{a}}$, T. Wübben ${ }^{\mathrm{b}}$, T. J. Balk ${ }^{\mathrm{b}}$, G. Dehm ${ }^{\mathrm{b}}$, L. Zhou ${ }^{\mathrm{c}}$, S. Günther ${ }^{\mathrm{d}}$, \\ E. Arzt ${ }^{\mathrm{b}}$, R. Imbihl ${ }^{\mathrm{c}}, \mathrm{R}$. Schlögl ${ }^{\mathrm{a}}$
}

\author{
${ }^{a}$ Department of Inorganic Chemistry, Fritz-Haber-Institute of the MPG, Faradayweg 4-6, 14195 Berlin, Germany \\ ${ }^{\mathrm{b}}$ Max-Planck-Institut für Metallforschung, Heisenbergstr. 3, D 70569 Stuttgart, Germany \\ ${ }^{c}$ Institut für Physikalische Chemie und Elektrochemie, Universität Hannover, Callinstr. 3 3a, \\ D 30167 Hannover, Germany \\ ${ }^{d}$ Department Chemie, Ludwig-Maximilians Universität München, Butenandtstr. 11 E, D-81377 München, Germany
}

${ }^{*}$ Corresponding author: e-mail ressler@fhi-berlin.mpg.de, phone +49 308413 3192, fax +49 3084134405

accepted 2005

\begin{abstract}
Thin copper films on silicon constitute model systems to investigate the influence of lattice strain on activity in heterogeneous catalysis. Thin copper films on silicon were investigated by ultraviolet photoelectron spectroscopy (UPS) to reveal the effect of strain in the copper films on the electronic structure of the surface. For cleaned and adsorbate-free surfaces, no effect of strain on the electronic structure was detected by UPS. Conversely, an oxygen-containing film exhibited a distinct effect of strain induced by cyclic heating and cooling on the electronic structure. Comparison with studies on a $\mathrm{Cu}$ single crystal under methanol oxidation reaction conditions revealed a characteristic hysteresis behavior in both the adsorbate structure and the catalytic properties of the metal surface. Hence, copper model systems that are suitable to unravel the correlation between strain and catalytic activity need to take the disordered microstructure of "real" copper catalysts into account. The present experiments reveal the correlation between surface restructuring and catalysis on the one side and the influence of lattice strain on either restructuring or the electronic structure of the surface on the other side.
\end{abstract}

Keywords: methanol synthesis, methanol steam reforming, strain, structure-activity relationships, copper, model system, real structure

\section{Introduction}

Copper based catalysts have been investigated for a long time in methanol chemistry, including methanol synthesis, steam reforming of methanol, and partial oxidation of methanol to formaldehyde. Growing experimental evidences indicate that the correlation between the activity of different copper catalysts and the specific copper surface area considerably deviates from a simple linear behavior. [1] Hence, various copper based catalysts may exhibit surfaces with different activities per unit metal surface area. [2 - 4] Possible explanations for this behavior include preferred exposure of different crystal surfaces, co-operative effects with the support (e.g. hydrogen spillover), electronic modification of copper by the support, modification of the electronic structure by strain, and copper sub-oxide formation. An unambiguous experimental verification of any of these hypotheses requires the ability to tailor the corresponding properties and to reveal their correlation with the catalytic activity. As it is difficult to vary a single structural property of a complex "real" system without affecting other relevant parameters, the corresponding experimental evidences for these explanations are still scarce. This also holds for a potential correlation of the microstrain in the copper particles and the catalytic activity. Theoretical calculations have shown that strained copper indeed exhibits significant changes in its electronic structure that can be relevant for adsorption and 
desorption reactions at the surface. [5] Experimental studies clearly show a non-linear correlation between $\mathrm{Cu}$ surface area and activity and suggest a positive effect of the increasing microstrain detected in the copper particles on the catalytic performance. [6 - 9] However, the structure-activity relationships described were obtained for "real" systems in which changes of other structural parameters such as crystallite size or chemical composition accompanied the variations in microstrain detected.

In order to disentangle the various structural parameters that determine the catalytic properties of a "real" system, appropriate model systems need to be designed. Selective investigations of the correlation between a particular structural feature and catalytic activity require model systems, which are more complex than single crystals, yet less complex than real catalysts. Thus, the model system sought has to bridge the "materials gap" between studies on ideal model systems and "real" industrial catalysts. The effect of strain on electronic or catalytic properties may be investigated both theoretically and experimentally by using pseudomorphic overlayers or surface alloys. In these systems, the lattice parameter of the metal under investigation, for instance copper, is changed by an epitaxial orientation on a differently sized metal such as $\mathrm{Cu} / \mathrm{Pd}$ or $\mathrm{Cu} / \mathrm{Au}$. Although, such samples are well defined and can be studied with conventional surface science techniques they are located on the "low-complexity" side of the materials gap and do not fully meet the requirements outlined above. Moreover, the ordered structure of some of these model systems is quite sensitive towards elevated temperatures while only one specific state of strain per sample type can be adjusted. Hence, different sample compositions are required to reveal the correlation between strain and catalytic activity. This again renders the deconvolution of strain and other factors such as electronic effects or ensemble effects difficult.

Model systems that permit varying the strain in a given sample and investigating the correlation between catalytic activity and different degrees of strain under reaction conditions would constitute a more promising approach. Two types of variably strained thin copper films deposited on a substrate are promising candidates for such model systems. First, a silicon single crystal wafer is used as a substrate and strain in the $\mathrm{Cu}$ film is induced by the different thermal expansion coefficients of $\mathrm{Cu}$ and $\mathrm{Si}$. [10 - 12] Because the residual strain exhibits a hysteresis depending on the temperature treatment, different states of strain can be obtained for a given temperature. Thin copper films on silicon can be readily employed in existing surface science equipment because no extra apparatus is needed to induce the strain in the copper film. Second, a polyimide foil is used as a substrate and the $\mathrm{Cu}$ film is strained mechanically with the help of a microtensile apparatus. [13 - 16] Because the level of complexity of supported polycrystalline thin films is located between those of simple single crystal surfaces and complex real catalysts, they constitute suitable model systems to bridge the materials gap.

Here, we introduce strained copper films on a silicon support as model systems for investigating the correlation between strain and electronic surface properties relevant for structure-activity relationships of methanol reactions on copper catalysts. The results obtained are compared to studies on a conventional $\mathrm{Cu}$ (110) single crystal model system used in the partial oxidation of methanol to formaldehyde.

\section{Experimental}

\subsection{Preparation of polycrystalline copper thin films on silicon wafers}

Polycrystalline copper films were grown by magnetron sputtering on (001)-oriented Si substrates (diameter: $50 \mathrm{~mm}$, thickness: $280 \mu \mathrm{m}$ ), which were coated on the front and back side with an amorphous $100 \mathrm{~nm}$ a-SiN $\mathrm{x}_{\mathrm{x}} \mathrm{a}-\mathrm{SiO}_{\mathrm{x}}$ bilayer to prevent $\mathrm{Cu}-\mathrm{Si}$ interdiffusion. After introduction into the ultra-high vacuum (UHV) deposition system, the substrates were $\mathrm{Ar}^{+}$-sputter cleaned $(105 \mathrm{~V} / 0.8 \mathrm{~A}$ for $1 \mathrm{~min}$ ) to remove possible surface contaminants. Film deposition was carried out using a self-constructed magnetron sputtering system (DAF at Max-Planck-Institute for Metal Research, Stuttgart) with a base pressure of $\sim 10^{-9} \mathrm{mbar}$. Prior to deposition, the copper target of $99.997 \%$ purity was degassed by pre-sputtering. Using a constant deposition rate of $41 \mathrm{~nm} / \mathrm{min}$, the desired film thickness between 35 and $500 \mathrm{~nm}$ was achieved by adjusting the deposition time accordingly. The samples were annealed in the vacuum chamber immediately after film deposition for $10 \mathrm{~min}$ at $600^{\circ} \mathrm{C}$ to saturate grain growth. Finally, the samples were cooled down to room temperature within 3 hours. This process route results in a tensile film stress at room temperature.

\subsection{Characterization of polycrystalline copper films on silicon}

The film stress during thermal cycling was studied by measuring changes in the curvature of the sample wafer using a laser scanning technique as described previously. [14 - 16] Focused ion beam (FIB) microscopy was used to investigate the microstructure of the films.

\subsection{UPS/XPS experiments on polycrystalline copper films on silicon}

\subsubsection{Experimental setup}

Ultraviolet photoelectron spectroscopy (UPS) and Xray photoelectron spectroscopy (XPS) were performed on sample pieces of approximately $8 \times 10 \mathrm{~mm}^{2}$ in size that were cut from original wafers with $50 \mathrm{~mm}$ in diameter. The latter were prepared at the same time and employing the same procedure as for the samples used for the stress measurements. The spectra were measured in a modified LHS/SPECS EA200 MCD system equipped with facilities 
for XPS (Al Ka $1486.6 \mathrm{eV}, 168 \mathrm{~W}$ power) and UPS (He I $21.22 \mathrm{eV}$, He II $40.82 \mathrm{eV}$ ). For the XPS measurements a fixed analyzer pass energy of $48 \mathrm{eV}$ was used resulting in a resolution of $1.05 \mathrm{eV}$ FWHM. The binding energy scale was calibrated using $\mathrm{Au} 4 \mathrm{f}_{7 / 2}=84.0 \mathrm{eV}$ and $\mathrm{Cu} \mathrm{2} \mathrm{p}_{3 / 2}=$ $932.67 \mathrm{eV}$. The base pressure of the UHV analysis chamber was $<1 \times 10^{-10}$ mbar.

\subsubsection{Sample cleaning and measurement}

Because the copper samples were exposed to air prior to the UPS/XPS experiment, the surface was covered by a copper oxide layer in the "as-received" state. In addition, carbon impurities were detected by XPS. Thus, some samples were exposed to $300 \mathrm{mbar}^{\mathrm{H}_{2}}$ in a pre-chamber of the spectrometer setup to reduce the surface oxide. The sample was heated to $250^{\circ} \mathrm{C}$ with a rate of $6 \mathrm{~K} / \mathrm{min}$ and kept at this temperature for 10 minutes, while the $\mathrm{H}_{2}$ atmosphere was replaced once. Subsequently, the chamber was evacuated and the sample was cooled down to room temperature. In another cleaning procedure, some samples were sputtered with $\mathrm{Ar}^{+}\left(5 \times 10^{-5}\right.$ mbar, $\left.1 \mathrm{kV}\right)$ for periods of time between $30 \mathrm{~min}$ and $2 \mathrm{~h}$. Since impurities dissolved in copper (especially oxygen) may segregate to the surface upon thermal treatment, and because after sputtering some contamination with argon was detected with XPS, every sputtering step was followed by heating to $500^{\circ} \mathrm{C}$ and cooling to room temperature $(6 \mathrm{~K} / \mathrm{min})$. After such a treatment, no Ar was detected by XPS. Following the cleaning procedure, the temperature-programmed UPS measurements were performed up to up to $500^{\circ} \mathrm{C}$ at a ramp rate of $6 \mathrm{~K} / \mathrm{min}$ followed by cooling to room temperature. During acquisition of a spectrum, temperature ramping was stopped. For both the heating and the cooling ramp, the same set of measurement temperatures was used, i.e. 25, 100, 200, 225, 250, $275,300,400$, and $500^{\circ} \mathrm{C}$. Each UPS spectrum was recorded in about 5 minutes.

\subsubsection{Data analysis}

After the cleaning procedures employed, the UPS spectra measured mainly correspond to that of copper metal. Only small changes in the spectra originating from the different degrees of strain in the copper film are expected. Hence, difference spectra had to be calculated to reveal the minute changes observable in the UPS spectra. For this procedure to result in a reliable correlation between strain and electronic surface structure, it is essential that the spectra used possess a common scale. Thus, much care was taken to keep the operation conditions as constant as possible. Despite an appropriate warm-up time of the UV lamp prior to the measurements and a continuous operation, slight changes in the lamp intensity with time were observed, that made it necessary to re-scale the spectra for evaluation. Therefore, a least-squares refinement procedure was used to scale pairs of isothermal UPS spectra to each other. On the one hand, this method will yield a zero difference in the experimental spectra (within the experimental noise) when no changes in the electronic structure of the surface are detectable. On the other hand, deviations in the experimental spectra used will result in observable features in the difference spectra, although shape and location of those features will be arbitrary and depend, for instance, on the energy range used.

\subsection{Selective methanol oxidation on a $\mathrm{Cu}$ (110) single crystal}

Methanol oxidation on a $\mathrm{Cu}$ (110) single crystal was conducted in a UHV system equipped with Auger electron spectroscopy (AES), low energy electron diffraction (LEED), photoelectron emission microscopy (PEEM), and quadrupole mass spectrometry (QMS) for temperatureprogrammed reaction spectroscopy (TPRS). The system was used as a continuous flow reactor in the pressure range from $10^{-7}$ mbar to $10^{-3}$ mbar. The base pressure of the system was $10^{-9}$ mbar. The QMS was differentially pumped. A ramping rate of $6 \mathrm{~K} / \mathrm{min}$ was used for the TPRS measurements. For the TPRS the sample was moved close to a cone shielding the QMS and assuring that in the mass spectrum only gas molecules were detected that had hit the sample surface once. Therefore, all conversion factors are equivalent to reactive sticking coefficients. The oxygen used during the TPRS experiments was introduced from a minican with 5.0 purity (Messer Griesheim). For methanol dosing the vapor over liquid methanol at room temperature was used. All gas dosing was performed using thermally isolated leak valves.

\section{Results and Discussion}

\subsection{Characterization of polycrystalline copper films on silicon}

Figure 1 shows the biaxial film stress as a function of the temperature for three copper films $(35 \mathrm{~nm}, 50 \mathrm{~nm}$, and $500 \mathrm{~nm}$ ). The stress detected results from the different thermal expansion between the silicon substrate and the copper film. It represents an integral quantity and may vary substantially from grain to grain, while the stress temperature evolution remains identical for subsequent thermal cycles. The typical microstructure of a polycrystalline $\mathrm{Cu}$ film grown on silicon is shown in Figure 2. The films exhibit a $\{111\}$ fiber texture with columnar grains. The lateral grain size is usually about twice the film thickness, i.e. thicker films have larger grains. With changing temperature, the copper film will first be deformed elastically (reversible), leading to a change of the interatomic distances (elastic strain). With increasing strain, plastic (irreversible) deformation sets in, compensating part of the temperature induced misfit. Plastic deformation of the film by dislocation motion and/or diffusional processes is responsible for the hysteresis observed in the stress-temperature curve in Figure 1. Thinner copper 


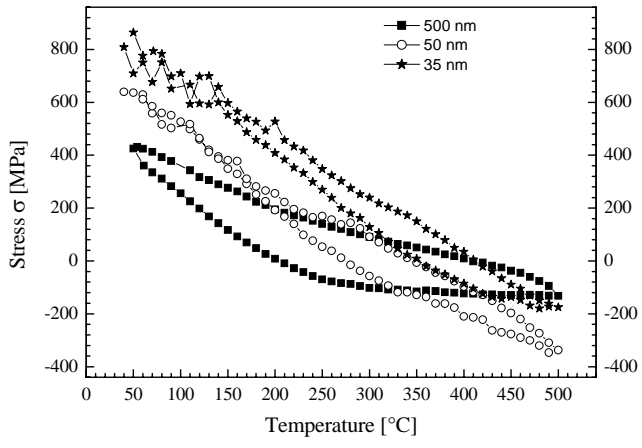

Figure 1: Stress-temperature curves for $35 \mathrm{~nm}, 50 \mathrm{~nm}$, and $500 \mathrm{~nm}$ thick polycrystalline copper films on a silicon substrate.

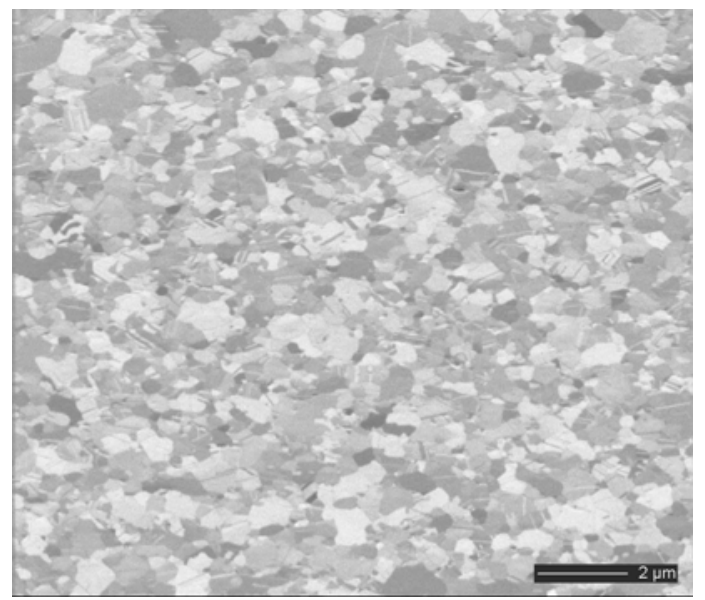

Figure 2: FIB image of a $100 \mathrm{~nm}$ thick polycrystalline copper film. The contrast between the grains changes depending on their orientation.

films show a higher degree of elastic (reversible) deformation, which results in a narrower stress-temperature hysteresis during thermal straining, with large values for biaxial tensile and compressive stresses at room temperature and at the maximum temperature, respectively. Conversely, thicker copper films exhibit a broader stress-temperature hysteresis during thermal cycling, but reveal lower stress values at room temperature and at the maximum temperature compared to thinner films. However, the broader hysteresis of thick copper films allows for larger differences in the stress at an intermediate temperature (e.g. $250^{\circ} \mathrm{C}$ ) between the heating and the cooling branch of a temperature cycle. Hence, thicker copper films on a silicon substrate should constitute more suitable model systems to investigate the effect of different degrees of stress in the copper film on electronic and catalytic surface properties.

\subsection{UPS/XPS investigation of polycrystalline copper films on silicon}

Three copper films of different thickness (500 nm, 50 $\mathrm{nm}$, and $35 \mathrm{~nm}$ ) on silicon substrates were studied with XPS (composition of sample surface) and UPS (surface band structure) during temperature-programmed experiments. Prior to the measurements, the $500 \mathrm{~nm}$ sample was reduced with hydrogen. However, significant amounts of carbon impurities were still detected after the treatment. Thus, the sample was subjected to alternating $\mathrm{Ar}^{+}$sputtering and heating-cooling cycles, which, after several iterations, resulted in reasonably low and constant levels of impurities. The $50 \mathrm{~nm}$ sample was sputtered once for $2 \mathrm{~h}$ before the hydrogen reduction step, which already resulted in a sufficiently low level of impurities.

Evaluation of the difference UPS spectra between corresponding down- and up-ramping measurements yielded minute features close to the noise level for both the 500 and the $50 \mathrm{~nm}$ copper films. These features were most pronounced at room temperature, suggesting that the amplitude of the changes is mainly determined by the time elapsed between the corresponding up- and down-ramping measurements. Hence, these features can be attributed to minor irreversible changes of the sample during thermal treatment, for instance, segregation of remaining impurities. However, the amplitude of the changes observed in the UPS spectra measured during thermal treatment of the $\mathrm{Cu} / \mathrm{Si}$ films was very small, indicating the overall excellent stability of the UPS/XPS equipment employed. Such a good stability is a prerequisite for the measurements described here. The effect of the strain in the bulk of the copper films on the electronic structure at the surface was impossible to distinguish from the irreversible changes detected in the UPS spectra.

Subsequently, an even thinner film of $35 \mathrm{~nm}$ was used to increase the elastic strain in the copper film. In contrast to the procedure described above, the copper surface was neither reduced nor sputtered to avoid possible changes in the local strain state by formation of point defects. Moreover, instead of a single heating-cooling cycle, two identical heating-cooling cycles with UPS measurements were conducted one after the other to be able to distinguish between irreversible effects (e.g. changes in the impurity level) and reversible effects such as the strain in the copper films that show a hysteresis behavior.

The UPS spectrum of the "as-received" $35 \mathrm{~nm}$ copper film on silicon showed no discernable features of the copper metal d-band because of coverage with copper oxide and impurities. Thus, the sample was subjected to one heatingcooling cycle to $773 \mathrm{~K}$ to enable possible reduction, desorption, and segregation processes for this temperature range before the actual UPS experiment. This "self-cleaning" treatment decreased the impurity level sufficiently to detect the copper d-band (Figure 3). Two broad features centered around $7.2 \mathrm{eV}$ and $11.3 \mathrm{eV}$ indicate the presence of residual oxide and of $\mathrm{OH}$ groups resulting from the self-cleaning process. 


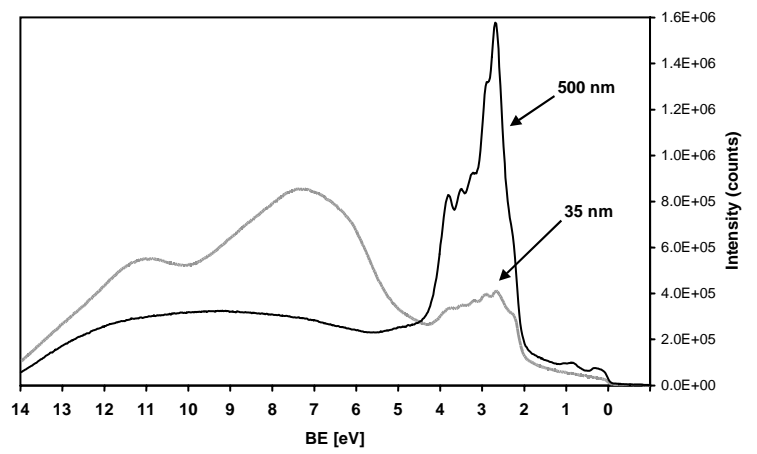

Figure 3: He I spectra of a $500 \mathrm{~nm} \mathrm{Cu}$ film after cleaning ( $\mathrm{H}_{2}$ reduction, several sputtering-heating-cooling cycles) and a $35 \mathrm{~nm}$ film after "self-cleaning" (one heating-cooling cycle). Coverage with oxygen and carbon impurities gives rise to the strong signal in binding energies (BE) from 6 to $12 \mathrm{eV}$.

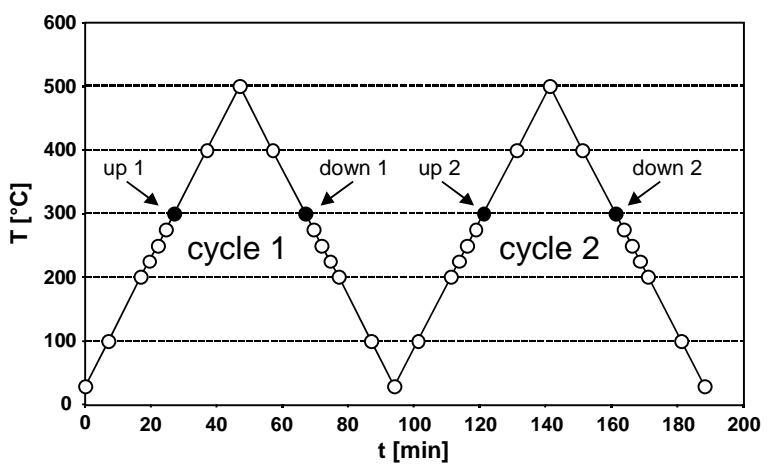

Figure 4: Schematic representation of the temperature program used for UPS measurements on the $35 \mathrm{~nm}$ sample. One set of four isothermal measurements $\left(300^{\circ} \mathrm{C}\right)$ is highlighted as an example.

From the UPS spectra obtained during two subsequent heating-cooling cycles (Figure 4), two types of isothermal difference spectra were calculated: (I) "Inter-cycle" difference spectra from corresponding measurements of the two cycles (i.e. up2-up1, down2-down1), (II) "intra-cycle" difference spectra from measurements during the heating and cooling branch within one cycle (i.e. down1-up1, down2-up2). Selected representative sets of difference UPS spectra are shown in Figure $5\left(200^{\circ} \mathrm{C}\right)$ and Figure $6\left(300^{\circ} \mathrm{C}\right)$. The inter-cycle difference spectra look very similar for all temperatures. Thus, the minor features detectable that correspond to irreversible changes in the copper films exhibit a linear dependence with time (i.e. about constant for constant time gaps). Conversely, the intra-cycle difference spectra change continuously as a function of temperature, with the difference spectra calculated for the two cycles being the same within the noise level. While the inter-cycle difference spectra should exhibit irreversible changes of the $\mathrm{Cu}$ film only, the intra-cycle data may represent a convolution of irreversible (time-dependent) and reversible hysteresis varia- tions in the copper film. Because the features in both types of difference spectra are qualitatively not the same, the comparison of inter- and intra-cycle data shows that reversible changes in the copper film can indeed be observed. The features in the intra-cycle difference spectra are most prominent (and most dissimilar to the inter-cycle difference spectra) at $300^{\circ} \mathrm{C}$, while the changes in the intra-cycle and intercycle difference spectra converge towards the beginning and the end of a cycle (room temperature and $500^{\circ} \mathrm{C}$ ).

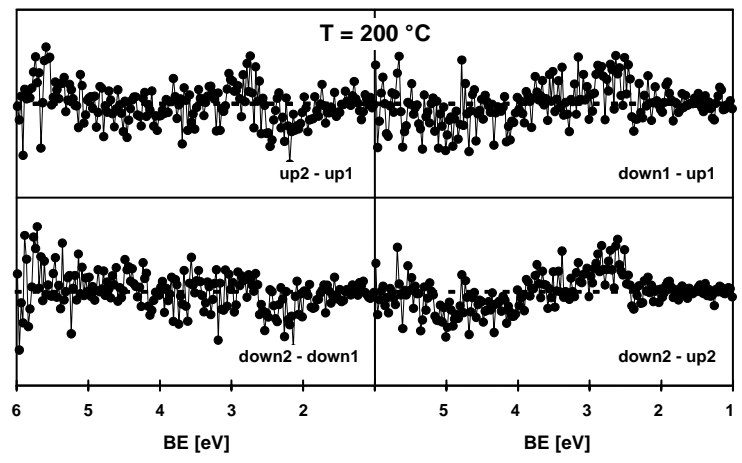

Figure 5: Inter-cycle (left) and intra-cycle (right) difference UPS spectra of the $35 \mathrm{~nm}$ thin copper film on a silicon substrate at $200^{\circ} \mathrm{C}$. The intra-cycle difference spectra exhibit a negative feature at $\sim 5 \mathrm{eV}$ and a small positive feature at $\sim$ $3 \mathrm{eV}$.

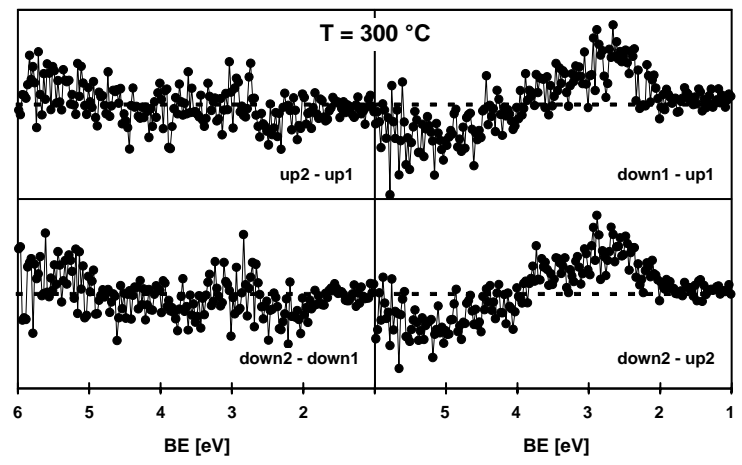

Figure 6: Inter-cycle (left) and intra-cycle (right) difference UPS spectra of the $35 \mathrm{~nm}$ thin copper film on a silicon substrate at $300^{\circ} \mathrm{C}$.

The results described show that in contrast to the thicker and cleaned $\mathrm{Cu}$ films (50 nm and $500 \mathrm{~nm}$ ), slight reversible changes could be detected in the UPS spectra of the $35 \mathrm{~nm} \mathrm{Cu}$ film indicating a certain hysteresis behavior of the electronic surface structure that may correlate with the evolution of strain in the film during thermal treatment. The different behavior observed in the UPS spectra of the three samples studied may be accounted for by the following explanations. (i) The $35 \mathrm{~nm} \mathrm{Cu}$ film (non-cleaned) shows a measurable strain at the film surface, while the strain at the surface of the thicker (cleaned) films is diminished by relaxation of the underlying bulk. (ii) The strain in the $\mathrm{Cu}$ films exhibits an effect on the electronic surface structure of the film, however the repeated sputtering and heating treat- 
ment of the thicker $\mathrm{Cu}$ films minimized the strain close to the $\mathrm{Cu}$ surface. (iii) The overall effect of the strain in the copper films on the electronic structure of a clean adsorbatefree surface is very small, which is in good agreement with corresponding theoretical calculation. Hence, the effect of the difference in the strain during heating and cooling of the cleaned $\mathrm{Cu}$ films (50 $\mathrm{nm}$ and $500 \mathrm{~nm}$ ) is not detectable by the UPS measurements performed.

Recent theoretical calculations have revealed that oxygen on the copper surface enhances the effect of the strain on the electronic structure of the surface. [9] According to the latter explanation it is proposed, that the reversible hysteresis effects detectable in the UPS spectra of the noncleaned $35 \mathrm{~nm}$ film result from the combined presence of strain and impurities (e.g. oxygen) that modify the electronic surface structure upon heating and cooling.

A positive correlation between catalytic activity and microstrain in copper metal particles has been reported for the steam reforming of methanol. [9] Although no bulk copper oxide phase was detectable under reaction conditions, water and carbon dioxide in the gas phase during the reaction make the presence of adsorbed or dissolved oxygen very likely. Moreover, it has been demonstrated recently for $\mathrm{Cu} / \mathrm{ZrO}_{2}$ catalysts for methanol steam reforming, that the catalytic activity correlates with the amount of oxygen in bulk of the copper nanoparticles. [17] Hence, it emerges that a catalytically relevant effect of strain in copper on the electronic structure of the surface may only be detectable in the presence of adsorbed or dissolved oxygen.

\subsection{Selective methanol oxidation on $\mathrm{Cu}$ (110) single crystals}

Methanol oxidation on a $\mathrm{Cu}$ (110) single crystal was investigated in the pressure range from $10^{-7}$ mbar up to $10^{-}$ ${ }^{3}$ mbar during temperature-programmed experiments. It can be seen from Figure 7 that the temperature of the maximum conversion is shifted to lower temperatures in the cooling branch of the experiment compared to the heating ramp resulting in a pronounced hysteresis. A correlation of the

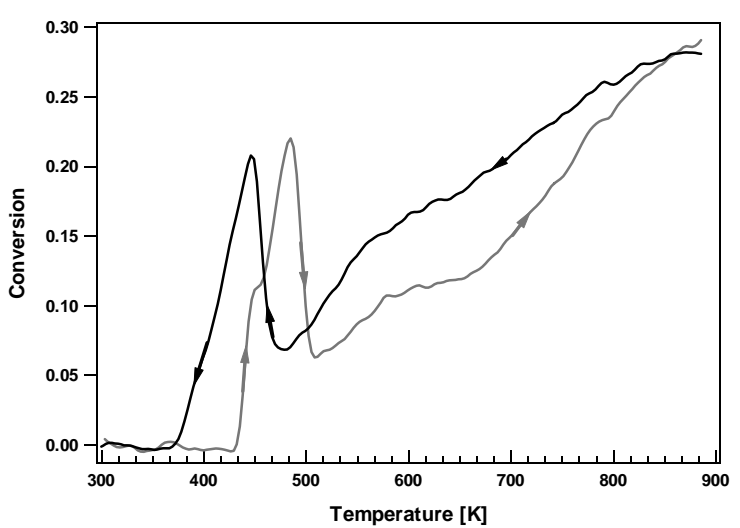

Figure 7: Methanol conversion on a $\mathrm{Cu}$ (110) single crystal during partial oxidation of methanol at $10^{-5}$ mbar total pressure. The observed hysteresis is attributed to differences in the adsorbate layer structure. catalytic data in the pressure range employed with a detailed analysis of the adsorbate layer by AES, LEED, and PEEM indicates that the surface reactivity is governed by the structures formed in the adsorbate layer. [18] This structure formation itself shows a pronounced hysteresis, i.e. it is dependent on the temperature pre-treatment of the system. Hence, with respect to investigating the influence of strain in copper under reaction conditions by a temperaturedependent hysteresis, it has to be taken into account that, at least in the lower pressure regime, activity and selectivity of the copper surface are governed by the structure of the adsorbate layer. The hysteresis in the adsorbate layer structure during heating and cooling will produce significantly different catalytic results for a given temperature, which may entirely mask the effect of strain on the electronic structure of the surface under reaction conditions. More details about the investigations of methanol oxidation on $\mathrm{Cu}(110)$ and the adsorbate structure formed as a function of reactant partial pressure will be reported elsewhere. [18]

\section{Conclusions}

The UPS results presented indicate, that the influence of strain in copper metal on the electronic structure of the copper surface may be difficult to detect for clean and adsorbate-free surfaces. Apparently, in order to verify a positive correlation between strain and activity as it has been observed for "real" catalysts, it will be inevitable to study strained copper model systems under reaction conditions (in situ). Appropriate adsorbates (e.g. oxygen) present on the surface under reaction conditions may be required to enhance the effect of the strain on the electronic structure and, thus, the catalytic activity. However, the $\mathrm{Cu}$ (110) model studies have shown, that under the reaction conditions employed the activity and reactivity of the copper surface is governed not only by a strain-modified electronic structure but also by the adsorbate layer. Moreover, the latter may also cause a hysteresis in the rate. Hence, any model system that requires cyclic heating and cooling to adjust the degree of strain in the copper will exhibit the inherent difficulty to disentangle the effect of strain and adsorbate layer on the catalytic properties obtained. Therefore, a model system is sought that permits studies under reaction conditions combined with the possibility to isothermally adjust different degrees of strain. The copper-polyimide system described above requires an additional microtensile apparatus to adjust the strain, which may be challenging to integrate into conventional set-ups used in surface characterization techniques. However, the advantage of being able to vary the strain isothermally over a large range makes this system a promising candidate for future model studies on the correlation between strain, surface electronic structure, and catalytic activity. The results presented show that copper model systems with a microstructure that closely resembles that of ideal copper metal will be of limited use to bridge the materials gap between "ideal" copper single crystals and "real" 
disordered copper particles supported on various metal oxides. Model systems employed to unravel the correlation between structure and activity of copper catalysts in methanol chemistry inevitably need to exhibit a certain degree of complexity to account for the disordered microstructure of "real” industrial copper catalysts.

\section{References}

[1] G. C. Chinchen and K. C. Waugh, Appl. Catal. 25 (1986) 101

[2] R. A. Hadden, P. J. Lambert and C. Ranson, Appl. Catal. A 122 (1995) L1

[3] K. C. Waugh, Catal. Lett. 58 (1999) 163

[4] M. Kurtz, H.Wilmer, T. Genger, O. Hinrichsen and M. Muhler, Catal. Lett. 86 (2003) 77

[5] S. Sakong and A. Groß, Surf. Sci. 525 (2003) 107

[6] M. M. Günter, T. Ressler, B. Bems, C. Büscher, T. Genger, O. Hinrichsen, M. Muhler and R. Schlögl, Catal. Lett. 71 (2001) 37

[7] M. M. Günter, T. Ressler, R. E. Jentoft and B. Bems, J. Catal. 203 (2001) 133

[8] J. B. Wagner, P. L. Hansen, A. M. Molenbroek, H. Topsøe, B. S. Clausen and S. Helveg, J. Phys. Chem. B 107 (2003) 7753

[9] B. L. Kniep, T. Ressler, A. Rabis, F. Girgsdies, M. Baenitz, F. Steglich and R. Schlögl, Angew. Chem. Int. Ed. 43 (2004) 112

\section{Acknowledgement}

The authors are grateful to the Deutsche Forschungsgemeinschaft, DFG, for financial support (SPP 1091, “Brückenschläge in der heterogenen Katalyse”).

[10] O. Kraft, M. Hommel and E. Arzt, Mat. Sci. Eng. A 288 (2000) 209

[11] M. Hommel, O. Kraft and E. Arzt, J. Mater. Res. 14 (1999) 2373

[12] M. Hommel and O. Kraft, Acta Mater. 49 (2001) 3935

[13] G. Dehm, T. J. Balk, H. Edongue and E. Arzt, Microel. Eng. 70 (2003) 412

[14] P. A. Flinn, D. S. Gardner and W. D. Nix, IEEE Trans. Electron. Devices 34 (1987) 689

[15] W. D. Nix, Metall. Mater. Trans. A 20 (1989) 2217

[16] R. M. Keller, S. P. Baker and E. Arzt, J. Mater. Res. 13 (1998) 1307

[17] A. Szizybalski, F. Girgsdies, A. Rabis, Y. Wang, M. Niederberger, and T. Ressler, J. Catal., submitted.

[18] L. Zhou, S. Günther, R. Imbihl, J. Catal., in press. 